\title{
Bulge Building with Mergers and Winds
}

\author{
By R. G. CA RLBER G
}

\author{
Department of Astronomy, University of Toronto, Toronto ON, M5S 3H8, Canada
}

\begin{abstract}
The gravitational clustering hierarchy and dissipative gas processes are both involved in the formation of bulges. Here we present a simple empirical model in which bulge material is assembled via gravitational accretion of the visible companion galaxies. Assuming that merging leads to a starburst, we show that the resulting winds can be strong enough that they selfregulate the accretion. A quasi-equilibrium accretion process naturally leads to the Kormendy relation between bulge density and size. Whether or not the winds are sufficiently strong and long lived to create the quasi-equilibrium must be tested with observations. To illustrate the model we use it to predict representative parameter dependent star formation histories. We find that bulge building activity peaks around redshift two, with tails to both higher and lower redshifts.
\end{abstract}

\section{Introduction}

Bulges are stellar dynamical pressure supported systems that generally have much higher surface brightnesses than galactic disks. They therefore have undergone more collapse than galactic disks, evidently with the angular momentum barrier removed. Galaxy merging is an inevitable process that redistributes any pre-merger stars into a physically dense, but phase density lowered, pressure supported distribution. Stellar dynamical mergers produce objects with flattenings largely unrelated to their rotation. In the presence of gas, merging is empirically associated with an often dramatic rise in star formation. These new stars that are formed in place almost certainly reflect the chemical history and the dynamical state of the growing bulge. This paper calculates some of the properties of bulges expected on the basis of merging with star formation of largely gaseous pre-galactic fragments.

The rate of major mergers can be calculated directly from the observed numbers of close pairs of galaxies. Remarkably, this is now an observational quantity for which there are measures from low redshift to the "U-dropout" population centred around redshift three. There are some substantial uncertainties in the various observational quantities which go into the merger calculation. The details of this calculation will become much more precise over the next few years as the evolution of the two-point correlation function becomes better determined.

Mergers are widely observed to induce an intense nuclear starburst. Theoretically this is at least partially understood (Barnes \& Hernquist 1992) on the basis that the strong dynamical interactions during a merger leads to a loss of angular momentum in a cool gas, helping to funnel it to build up a dense central gas reservoir from which stars form at astonishing rates in a starburst. Starbursts in turn develop winds which we suggest can lead to the accretion being a self-regulating, although this is dependent on the ram pressure and duration of the wind. Moreover, self-regulating accretion can lead to quasi-equilibrium star formation in the bulge which can lead to some of the observed regularities of bulge properties with mass or size. At this stage the details of this picture are speculative, but are open to observational refinement, which helps motivate the calculations presented here.

Other papers presented at this meeting describe in detail the properties of bulges. Here we take the properties of "classical bulges" to be roughly as follows (e.g. Wyse Gilmore \& Franx 1997). 
- Bulges follow the "Kormendy relations", that is, the characteristic surface brightness correlate strongly with the scale radius (Kormendy 1977, De Jong 1996, Pahre, Djorgovski \& De Carvalho 1995).

- The flattening of the figure of bulges is approximately consistent with their rotation (Davies et al. 1983).

- Bulge stellar populations are predominantly old, although there are well-documented cases of relatively young bulges.

- Bulges have a mass-metallicity correlation.

A useful model for bulges should be able to provide a physical origin for these properties.

The paper has three main sections. In $\S 2$ we discuss the empirically determined rate at which mergers occur as a function of redshift. Then, in $\S 3$, we discuss star burst winds, and the effects those winds will have on accreting gas. Section 4 pulls these two together in some specific model calculations.

\section{Merger Rate Measures}

A host galaxy has a number of near neighbors within radius $r$ and pairwise velocity $|v|$ far above the mean density $n_{0}$ (in proper co-ordinates),

$$
N(<r,<v)=4 \pi n_{0}(1+z)^{3} \int_{0}^{v} \int_{0}^{r} \xi(r \mid z) f(v \mid z) r^{2} v^{2} d r d v,
$$

where $\xi(r \mid z)$ and $f(v \mid z)$ are the redshift dependent two point correlation and velocity distribution functions, respectively. We have made the important assumption that the distribution of pairwise velocities is constant over the separations of interest. This is not true in general, but is sufficient for our application to the relatively small scales, $r \leq 20 \mathrm{kpc}$, that are of interest for merging. To calculate merger rates we need estimates of the correlation function on small scales, the pairwise velocity dispersions, and the mean time for a merger to occur within this volume.

\subsection{Close Pairs and the Correlation Function}

The galaxy-galaxy correlation function is accurately modeled as a power law, $\xi(r)=$ $\left(r_{0} / r\right)^{\gamma}$. The reliability of this power law on scales less than about $100 h^{-1} \mathrm{kpc}$ relevant to galaxy merging is discussed in detail for the SSRS2 (Patton et al. 1999a) and CNOC2 samples (Patton et al. 1999b). These papers support three important conclusions. First, the power law extrapolation of the correlation function to $20 \mathrm{~h}^{-1} \mathrm{kpc}$ is consistent with the density of pairs measured inside this radius. Second, the $\mathrm{R}$ band luminosity function of galaxies in $20 h^{-1} \mathrm{kpc}$ pairs is consistent with being drawn from the field luminosity function. This property allows the fully general correlation function, $\xi\left(r, v_{\|}, v_{\perp}, L_{1}, L_{2}\right)$, to be factored into a luminosity function, and a spatial and kinematic correlation function, $\phi\left(L_{1}\right) \phi\left(L_{2}\right) \xi(r) f(v)$ where we drop the distinction between velocities along the line of separation, $v_{\|}$, and perpendicular velocities, $v_{\perp}$ (Peebles 1980). The luminosity factorization glosses over the various lines of evidence (Carlberg et al. 1998, Loveday et al. 1995) that the theoretically expected weak increase of correlations with galaxy mass does exist in the correlations. However, this relatively small effect cannot yet be detected in the current samples of close pairs which have not yet broken through the barrier of 100 pairs. The third important result is that there is morphological evidence that $r \leq 20 h^{-1} \mathrm{kpc}$ pairs are indeed interacting at a level that indicates that these are high probability mergers-to-come. The $20 h^{-1} \mathrm{kpc}$ scale is also chosen such that the galaxies are not so strongly interacting that their luminosities, morphologies and colors bear little resemblance to their unperturbed values. 


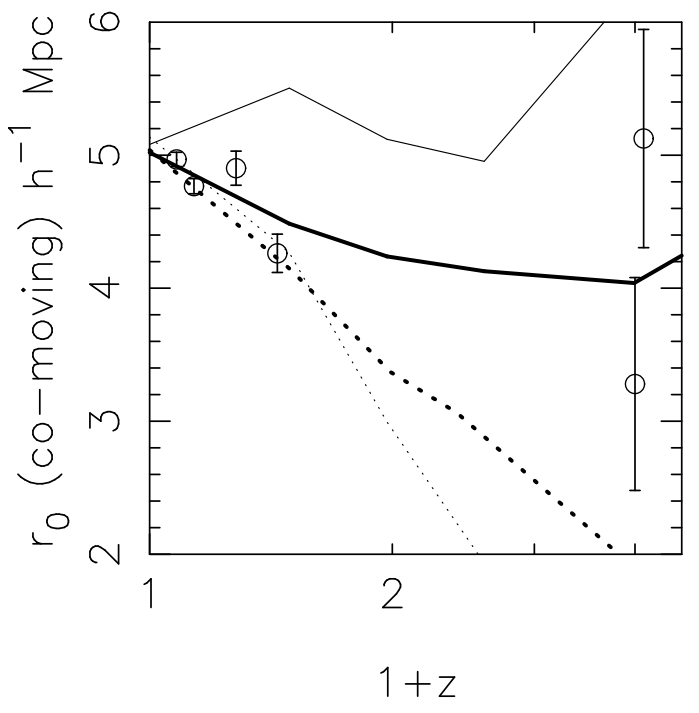

FiguRE 1. Measured co-moving correlation lengths as a function of redshift. The points are the LCRS at low redshift, CNOC2 at intermediate redshift, and the Giavalisco et al. (1998) close pairs and the "box counts" of Adelberger et al. (1998) at redshift 3. The lines are for the correlation lengths measured in simulations (Colin, Carlberg \& Couchman 1997) for galaxy halos (solid lines) and the mass field (dashed), with $\Omega_{M}=0.2$, and $\Omega_{\Lambda}=0$ (heavy) or $\Omega_{\Lambda}=0.8$ (light).

The volume integral of the power law correlation function, $\xi(r \mid z)=\left(r_{0}(z) / r\right)^{\gamma}$, is,

$$
4 \pi \int_{0}^{r} \xi(r \mid z) r^{2} d r=\frac{4 \pi}{3-\gamma}\left(\frac{r_{0}(z)}{r}\right)^{\gamma} r^{3} .
$$

The redshift dependence of the average density inside a $r=20 h^{-1} \mathrm{kpc}$ neighborhood around a galaxy is estimated using the preliminary CNOC2 correlation $\gamma=1.8$,

$$
r_{0}(z)=5.15(1+z)^{[1-(3+\epsilon) / \gamma]} h^{-1} \mathrm{Mpc} \text { (co-moving), }
$$

where $\epsilon=-0.6 \pm 0.4$ (Carlberg et al. 1998). Using this in Equation 2.2 we find that the integrated density inside $20 h^{-1} \mathrm{kpc}$ is $1.56[(1+z) / 1.3]^{-\epsilon} n_{0} \mathrm{Mpc}^{3}$ (proper units). In Figure 11 we show the co-moving correlation length as a function of redshift. We also plot the correlations of the galaxy mass halos and the particles in simulations (Colin, Carlberg \& Couchman 1997). It should be noted that there is a relatively good understanding of why the halo correlation function shows relatively little evolution (Carlberg 1991, Baugh, et al. 1998). Observationally it is currently acceptable to take $r_{0}$ to be fixed over this redshift interval, or, $\epsilon=-1.2$ for a $\gamma=1.8$ power law.

\subsection{Pairwise Velocities}

The CNOC2 velocity distribution function, $f(v)$, at small scales is acceptably modeled as a Gaussian, although an exponential also provides a similar quality fit (Carlberg et al. 1998). We will take the velocity to be isotropic, $\sigma_{3}=\sqrt{3} \sigma_{z}$, where $\sigma_{z}$ is the velocity dispersion measured along the line of sight. Redshift surveys can be used to measure $\sigma_{z}$. At separations of about $1 h^{-1} \mathrm{Mpc}$ the the pairwise velocity dispersion in the redshift direction is about $300 \mathrm{~km} \mathrm{~s}^{-1}$ (e.g. Davis \& Peebles 1983 , Marzke, Geller, Da Costa \& Huchra 1995, Carlberg et al. 1998), constant with redshift over the $z \leq 0.5$ range. If the critical velocity to merge is taken as about $220 \sqrt{2}$ 
$\mathrm{km} \mathrm{s}^{-1}$ then the fraction of close pairs with sufficiently low velocities to merge is merely $5.1 \%$. This does not accord well with the impression that most close pairs have such large tidal tails that they are almost certainly doomed-to-merge pairs (Toomre \& Toomre 1972, Tooomre 1977). In a non-merging, equilibrium distribution the pairwise velocity declines as $\sigma_{p}^{2} \propto r^{2-\gamma}$, where $\gamma$ is the slope of the power law correlation, The one dimensional pairwise velocity dispersion at $20 \mathrm{kpc}$ is therefore about $190 \mathrm{~km} \mathrm{~s}^{-1}$.

The dynamical details of pair mergers in a cosmological setting that includes the tidal fields of surrounding structure have not been studied in detail (but see Carlberg \& Couchman 1989), such that one of the best estimates of the critical velocity to merge remains Aarseth and Fall's (1980) value of $v_{m g}=1.2 \sqrt{2} v_{p}$, where $v_{p}$ is the "parabolic" velocity, $v_{p}=f_{p} v_{c}$, at the orbital pericenter, where $f_{p}$ is at least $\sqrt{2}$ (for a point mass). This "parabolic" velocity is that for those pairs assured to merge, in the absence of tidal fields (Toomre \& Toomre 1972, Aarseth \& Fall 1980). For $f_{p}=\sqrt{2}$ the fraction of the the close pairs with velocities low enough to merge is $27 \%$ but this rises quickly with increasing $f_{p}$, becoming $41 \%$ and $54 \%$ at $f_{p}=\sqrt{3}$ and 2 , respectively. We will use an $f_{m g}=0.5$ but recognize that this number is both empirically and theoretically uncertain.

\subsection{Merger Times of Close Pairs}

As a reference timescale for merging we start from our reference radius of $20 \mathrm{kpc}$ where the time for a circular orbit is $0.62 \mathrm{Gyr}$ at a speed of $220 \mathrm{~km} \mathrm{~s}^{-1}$. In detail the rate of inflow through the $20 \mathrm{kpc}$ depends on mass and the orbital details, so we use a merger timescale at our reference radius of $20 \mathrm{kpc}$ of $0.3 \mathrm{Gyr}$ (Barnes \& Hernquist 1992 , Dubinski, Mihos \& Hernquist 1999). We use our estimate that $f_{m g}=0.5$ of all $20 \mathrm{kpc}$ pairs have pairwise velocities less than the critical velocity for merging. If the pairwise velocities and substantially higher than the critical velocity for merging then the merging fraction drops nearly as $v^{3}$, which is such a dramatic change that it should be testable via the morphology of galaxies in close pairs.

\subsection{Estimated Merger Rates}

Combining our estimates of clustering, pairwise velocities and the available understanding of the dynamics of merging, we find that the specific merger rate is $2.4[(1+$ $z) / 1.3]^{-\epsilon} n_{0} \mathrm{Mpc}^{3} \mathrm{Gyr}^{-1}$. If we take $n_{0}$ as being the CNOC2 galaxy number density to $0.21 L_{*}$ adjusted to $0.1 L_{*}, n_{0} \simeq 1.1 \times 10^{-2} \mathrm{Mpc}^{-3}$ (co-moving) the merging event rate for galaxies above our minimum mass is $1.3 \times 10^{-2}(1+z)^{-\epsilon} \mathrm{Gyr}^{-1}$. The rate of accretion of pre-merger stars onto galaxies as a result of merging is relatively slow. The time scale is $L_{*} / \mathcal{R}_{L} \simeq 60 \mathrm{Gyr}$, or about 5 Hubble times at $z=0.3$. This result is based on the directly visible galaxies, $L \gtrsim 0.21 L_{*}$, which contain about $80 \%$ of the total stellar luminosity galaxies. This relatively low rate of accretion of visible galaxies relative to the Hubble times continues on to the $z \simeq 3$ regime (Giavalisco et al. 1998, Adelberger et al. 1998), where the number densities and the co-moving correlations are similar to those observed for present day galaxies. That is, for $q_{0}=0.1$ their data indicate a density of $\mathcal{R}<25.5$ mag Lyman break galaxies of $n_{0} \simeq 2.2 \times 10^{-3} \mathrm{Mpc}^{-3}$ and a co-moving correlation length of $5 h^{-1} \mathrm{Mpc}$. The self-event rate of this population is $2.0 \times^{-2} \mathrm{Gyr}^{-1}$, only 3.8 times that at $z \simeq 0.3$. Since the cosmic time at $z \simeq 3$ is about $20 \%$ of that at $z=0.3$ the relative impact on the hosts of these self-mergers is small adding perhaps $5-10 \%$ more mass over the entire $z=0$ to 3 range. For the lower luminosity galaxies (about 2 magnitudes fainter than those with spectroscopic redshifts) inferred to be in this redshift range from the HDF the volume density is about a factor of 20 higher, but the implied cross- 
correlation length is about a factor is about a factor of $\sqrt{3}$ smaller (Steidel et al. 1999), where we assume that the cross-correlation depends on the product of the relative biases. This implies that the high-low luminosity merger rate is about $0.5 \mathrm{Gyr}^{-1}$ which is large enough to build a galaxy over a $z \simeq 1-4$ interval. In the intermediate redshift range the $M \gtrsim 0.2 M_{*}$ galaxies cannot self-merge to significantly alter the mass function. At higher redshift the lower luminosity Lyman break galaxies rise very steeply in number, $\alpha \simeq-1.8$ (Steidel et al. 1999). These large numbers completely change the situation, allowing their mergers to substantially alter galaxy masses.

\section{Starburst Winds}

There is a remarkably simple physical description of what happens when star formation is rapid in a relatively small volume. The inevitable outcome is a very strong wind. Chevalier \& Clegg (1985, hereafter CC) simplify the situation to the equilibrium solution of mass injection at a rate $\dot{M}$, with accompanying energy injection, $\dot{E}$, in a sphere of radius $R$. Since it turns out that the wind velocities exceed $1000 \mathrm{~km} \mathrm{~s}^{-1}$, gravity can be ignored in a first approximation. CC provide a full solution at all radii. Here we are mainly interested in the asymptotic solution at large radii, where we cast the CC solution in terms of the terminal wind velocity, $u$ and the mass injection rate, $\dot{M}_{w}$,

$$
\rho_{w}=\frac{\dot{M}_{w}}{4 \pi u r^{2}}
$$

This wind produces a ram pressure of,

$$
P_{w} \equiv \rho_{w} u^{2}=\frac{u \dot{M}_{w}}{4 \pi r^{2}}
$$

where $R$ is the size of the region into which the mass and energy are injected.

As representative numbers we will take $R=10^{21} \mathrm{~cm}$, about $1 / 3 \mathrm{kpc}$, and $\dot{M}_{w}=$ $10^{27} \mathrm{~g} \mathrm{~s}^{-1}$, about $15 \mathrm{M}_{\odot} \mathrm{yr}^{-1}$, approximately the mass injection rate expected during a burst of star formation of $1500 \mathrm{M}_{\odot} \mathrm{yr}^{-1}$. We follow CC and use $u=2000 \mathrm{~km} \mathrm{~s}^{-1}$. In the central region,

$$
\rho_{w} \simeq 0.296 \frac{\dot{M}_{w}}{u R^{2}}
$$

or $1.5 \times 10^{-24} \mathrm{~g}$, or a number density of about $1 \mathrm{~cm}^{-3}$.

The cooling time, $t_{\text {cool }}=3 k T /(n \Lambda(T))$, at $T=10^{8} \mathrm{~K}$ where the cooling rate is about $\Lambda \simeq 3 \times 10^{-23} \mathrm{~cm}^{3} \mathrm{~s}^{-1}$ is about $4 \times 10^{7} \mathrm{yr}$. The flow time across the region is only $1.6 \times 10^{5} \mathrm{yr}$, so the hot wind does not have time to cool. Denser regions in pressure equilibrium will cool more quickly so that the ISM is unstable and bound to consist of the hot wind phase and one or more cool phases. Many of the aspects of this situation are discussed in Ikeuchi \& Norman (1991).

\subsection{Ram Pressure Stripping}

A major objective of this paper is to estimate the ram pressure stripping of the hot wind on infalling objects. We calculate the effects of ram pressure stripping, but note that transport processes, such as turbulence and heating, could help to increase the rate of gas removal (Nulsen 1982). The calculation proceeds in a series of steps. First, we derive for our specific case the fairly standard results that the wind will have a very high momentum flux. The wind has a sufficiently low density that it will move out before cooling. The infalling objects are taken to be angular momentum conserving, but maximally dissipated disks in dark halos, approximated as truncated Mestel disks. 
The fractional stripping can be easily estimated for these objects. The strength of the starburst wind is calculated under the assumption that star formation is occurring on timescale comparable to the crossing time of the bulge. Bringing these elements together gives an expression for the fractional mass of an infalling object which succeeds in joining the bulge, Equation 3.16 .

The ridge line of Kormendy's relation is $\mu_{B}=3.02 \log r_{0}+19.74 \mathrm{~B}$ mag $\operatorname{arcsec}^{-2}$ (Kormendy 1977, De Jong 1996), where $\mu_{B}$ is the B band surface brightness and $r_{0}$ is the bulge scale radius in kpc. For a constant mass-to-light ratio this translates to $\rho \propto r_{0}^{-2.2}$. This density radius relation would be slightly weakened if we allowed for a decrease in mass-to-light for lower luminosity systems. The implied densities are high enough that bulges are self-gravitating. If the rate of infall is, on the average, regulated by the starburst winds, then the fact that the infall rate is very insensitive to the mass of the host implies that the characteristic radius and density of the bulge will scale roughly as $\rho \propto R^{-2}$, as in Equation 3.14. This relation is roughly the ridge line of the Kormendy relation. This is physically easy to understand. The total bulge star formation rises as the bulge gas density, $\rho_{b}$, to the $3 / 2$ power. For a given accretion rate. a rise in bulge density will increase the SFR, and hence the outgoing wind, which temporarily reduces accretion, allowing the gas density to be reduced.

The ram pressure rises as $r^{-2}$ with decreasing radius. At $R$, the outer radius of the star forming volume, the surface density below which stripping occurs is rises to its maximum,

$$
\Sigma \frac{V_{c}^{2}}{R}=\frac{u \dot{M}_{w}}{4 \pi R^{2}}
$$

where $V_{c}$ is the circular velocity of the incoming gas in its dark halo. For the starburst numbers above, $\Sigma=0.16 \mathrm{~g} \mathrm{~cm}^{-2}$ is the maximum surface density that can be blown away via ram pressure alone. For comparison. the central surface density of a disk galaxy is about $1 \mathrm{~g} \mathrm{~cm}^{-3}$. We conclude that the effects of this wind would be significant even on disk like that of the Milky Way if it were completely gaseous.

If we collapse the gas inside an isothermal halo with a velocity dispersion $\sigma_{s}$ and an angular momentum parameter $\lambda \simeq 0.05$ to centrifugal equilibrium its surface density increases by about a factor of $10^{2}$ over that of the projected isothermal halo,

$$
\Sigma(d) \simeq 10^{2} \Omega_{b} \frac{\sigma_{s}^{2}}{2 d G}
$$

where $d$ is the radial co-ordinate in the disk. For our typical case, $\Omega_{b} \simeq 0.01$, $H=100 \mathrm{~km} \mathrm{~s}^{-1} \mathrm{Mpc}^{-1}$ we find that this Mestel disk has a surface density profile,

$$
\Sigma(d) \simeq 0.7\left(\frac{\sigma_{s}}{100 \mathrm{~km} \mathrm{~s}^{-1}}\right)^{2}\left(\frac{10^{21} \mathrm{~cm}}{d}\right) \mathrm{g} \mathrm{cm}^{-2} .
$$

The total mass in a Mestel disk of the above form diverges. If we cut the disk off at the radius of the last orbit that can have come from the outermost virialized part of the halo we can estimate a total mass. The halo is virialized inside approximately $r_{200}$, the inside of which the mean density is $200 \rho_{c}(z)$, where $\rho_{c}(z)=3 H(z)^{2} /(8 \pi G)$. For an isothermal sphere, $M(r)=800 / 3 \pi \rho_{c}(z) r_{200}^{2} r$. The isotropic velocity dispersion that maintains this sphere in equilibrium is, $\sigma_{1}^{2}=400 / 3 \pi G \rho_{c}(z) r_{200}^{2}$. With the definition of $\rho_{c}(z)$ we find that for the isothermal sphere,

$$
r_{200}=\frac{\sqrt{2}}{10} \frac{\sigma_{s}}{H(z)} .
$$

This is a physical (proper) distance. In this potential the circular velocity is $V_{c}=\sqrt{2} \sigma$.

The total gas mass in the disk (assuming that there are few stars) is $200 \rho_{c} \Omega_{b} \frac{4}{3} \pi r_{200}^{3}$, 
or,

$$
M_{m}=\Omega_{b} \frac{2 \sqrt{2}}{10 G H(z)} \sigma_{s}^{3},
$$

which is needed to find $\sigma_{s}$ which is required in the evaluation of $d / r$ for stripping below, Equation 3.16.

\subsection{Starburst Rates}

Both empirical evidence and theoretical considerations suggest that the timescale for star formation should be proportional to the local dynamical timescale (Lehnert \& Heckman 1996, Kennicutt 1998). The available data point to a relation of $t_{s f r} \simeq R / v_{b}$, where $v_{b}$ is the local circular velocity, which in the case of a stellar bulge may be due to self-gravity, not the dark matter background. Therefore $\dot{M}_{S F R}=M v_{b} / R$, and we will take the wind as $\dot{M}_{w}=\epsilon_{w} \dot{M}_{S F R}$. The mass can be expressed as $M=4 \pi / 3 \rho R^{3}$, where $\rho$ refers to the gas density.

The wind that results from this starburst has a ram pressure,

$$
P_{w}=\epsilon_{w} v_{b} u \rho \frac{4 \pi}{3} \frac{R^{2}}{r^{2}} .
$$

\subsection{Self-regulating Starbursts}

The starburst wind will blow away infalling surface densities smaller than,

$$
\Sigma \frac{V_{c}^{2}}{r}<\epsilon_{w} v_{b} u \rho \frac{4 \pi}{3} \frac{R^{2}}{r^{2}} .
$$

In equilibrium this leads to a balance of the bulge which creates the wind and the and infall,

$$
\rho R^{2} \simeq \Sigma \frac{3}{4 \pi \epsilon_{w} u} \frac{V_{c}^{2} r_{200}}{v_{b}} .
$$

If bulges are to be self-gravitating, then $v_{b} \gtrsim V_{c}$, and generally they are found to have circular velocities comparable to those of the disk. The important thing to note is that the quantity $\rho R^{2}$ is completely determined by the starburst, whereas the RHS is completely determined by the physics of infall.

For Mestel disks derived from satellite halos of $\sigma_{s}$, and $\sigma_{b}^{2}=2 \pi G \rho R^{2}$ are stripped at radii beyond,

$$
\frac{d}{r}>\frac{\sigma_{s}^{2}}{\sigma_{h}^{2}} \frac{3}{4 \epsilon_{w}} \frac{v_{b}}{u} 10^{2} \Omega_{b},
$$

For typical numbers we take $\sigma_{s}=\sigma_{h} / 2$ and $\epsilon_{w}=10^{-2}$. We find stripping at $d / r>1.3$, which for $r=R$, and $R \simeq 1 / 3 \mathrm{kpc}$ is $99.5 \%$ of the disk mass.

The equation for stripping of Mestel disks can be used to predict the rate of successful accretion in the presence of a starburst. We simply multiply $M_{m}$ with the ratio of $d / r$ for stripping. The accreted mass is simply

$$
M_{\text {accrete }}=\frac{d}{r} \frac{R}{10 r_{200}} M_{m} .
$$

\section{Realizations of Bulge Formation Histories}

Combining merging rates and stripped fractions to build bulges with redshift is now straightforward. To illustrate the model we do a simple Monte Carlo simulation. We start the simulation at when the universe is about $0.5 \mathrm{Myr}$ old, prior to significant galaxy 


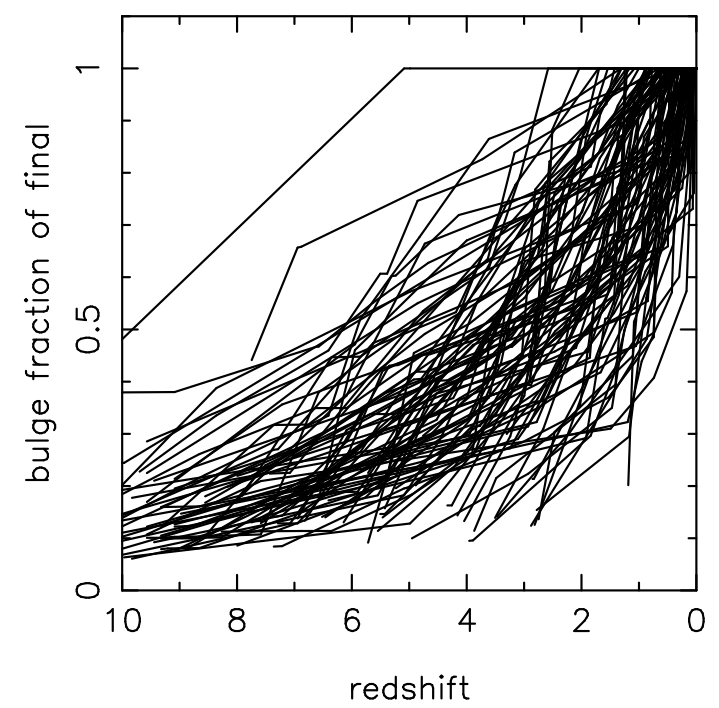

FiguRE 2. Mass redshift history for 100 realizations of bulge building. The calculation assumes that suitable pre-galactic objects, disks in dark halos, are available at the beginning of the calculation.

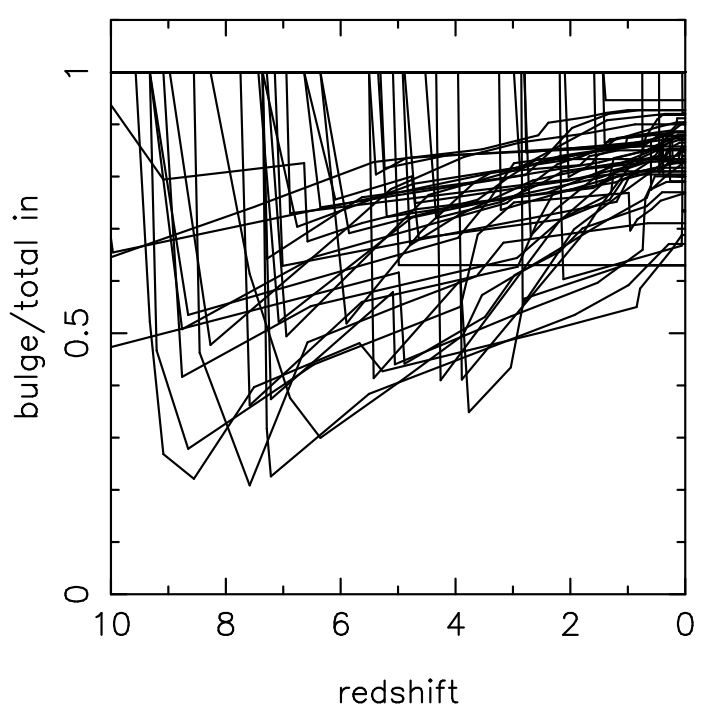

FigURE 3. Fraction of infalling gas mass successfully accreted versus redshift for the 100 realizations of bulge building.

creation or merging. We adopt an $H_{0}=65, \Omega_{M}=0.2, \Lambda=0$ cosmology, although the results are not very sensitive to the precise choice of cosmology. The mergers occur at a rate independent of mass at at a rate $\mathcal{R}_{n}(1+z)^{m}$. Time is divided into 0.5 Myr intervals and the probability of an accretion event is the rate per unit time multiplied with the time interval. An accretion event occurs if a $[0,1]$ random number generator produces a number less than this probability. The accreted objects begin with a mass drawn from $\phi(M) \propto M^{\alpha} e^{-M}$, where the normalizing constants are unity. This therefore assumes that the Mestel disks in their dark halos are largely present when the calculation is turned on. 
TABLE 1. Merger-Wind Simulation Parameters

$\begin{array}{lrr}\text { Parameter } & \text { Symbol } & \text { Default Value } \\ \text { Minimum satellite mass } & M_{\min } & 0.1 \\ \text { Mass function slope } & \alpha_{M} & -1.8 \\ \text { Current merger rate } & \mathcal{R}_{n} & 0.01 \\ \text { Merger-redshift index } & m & 1.2 \\ \text { Gas fraction } & \Omega_{g a s} & 0.01 \\ \text { Bulge size } & R_{b} & 10^{21} \mathrm{~cm} \\ \text { Wind duration } & T_{w} & 10^{8} \mathrm{yr} \\ \text { Mass loss efficiency } & \epsilon_{w} & 0.01\end{array}$

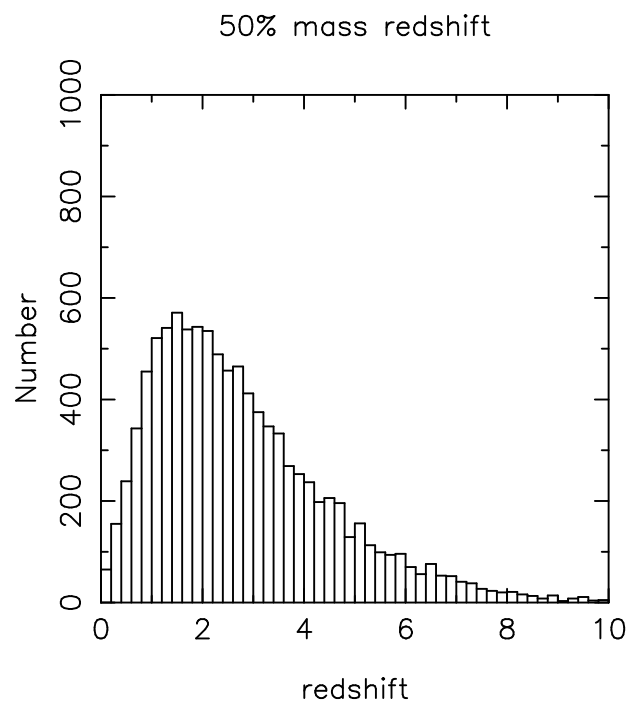

Figure 4. Redshift of $50 \%$ mass assembly for 10000 realizations of the standard model.

Clearly this is not accurate at large redshift, but is arguably a useful assumption over the redshift zero to about four range (Steidel et al. 1999). There is no presumption that the characteristic mass is as large as that of a full galaxy today, however the characteristic mass does need to be comparable to a bulge mass, since we find that the final masses are distributed around unity, the characteristic mass of the infalling objects. The wind is assumed to blow uniformly for a duration of $T_{w}$ from the previous merger. If a new accretion event is generated during this interval, then the mass of the incoming satellite is reduced according to the stripping equation. The parameters and their default values are outlined in the following table.

In detail there are many (mostly minor) complications that are swept under the rug here. The model is quite naive in that we assume that there is a ready supply of gas containing companion galaxies with roughly a galaxy mass distribution. Observation seems to support this as being true from redshift zero to about four, which covers most of the activity here. Mergers wouldn't do much at all if $\alpha \gtrsim-1.2$, as is observed at low redshift. We have chosen a steeper $\alpha$ to both mimic the increase in gas content with decreasing mass, and, to take into account that $\alpha$ does become steep in the redshift 3-4 range (Steidel et al. 1999). The gas content of galaxies should likely vary with redshift, whereas we have simply taken them to be all gas. Likely the pairwise velocity dispersion 


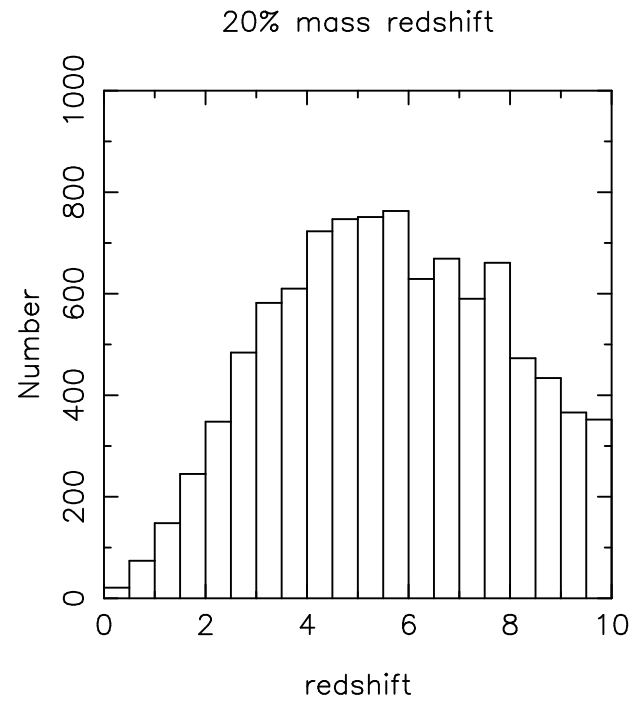

Figure 5. Redshift of $20 \%$ mass assembly for 10000 realizations of the standard model. Comparison with Figure 4 emphasizes the very large spread in redshifts of formation.

decreases somewhat with increasing redshift, which will diminish the fraction of low redshift pairs that merge. Overall the effect of all of these things would be to decrease the importance of merging at below redshift one. The natural tendency of the model is to have little activity at low redshift anyway, so the basic character of the results should not depend on these simplifications. In any case, the main purpose of this "toy" star formation history is to examine the basic viability of the model, not to fine tune the parameters.

Overall, we find several interesting results. First, the mass buildup predicted by this simple model seems to be very roughly in accord with the requirements of "classical" bulges. Figure 1 shows that About $10 \%$ are more than $50 \%$ formed at redshift 5 . But, about $3 \%$ are only half formed at redshift 0.5 . Although the median time of half assembly is a reassuring redshift of about two, there is a tremendous spread of formation times. The redshifts of $20 \%$ assembly are shown in Figure 5 .

The standard wind has a lifetime of $10^{8}$ years, which in many cases limits infall to about $2 / 3$ of what it would normally be, Figure 3 . The limitation of infall would help drive bulges towards the Kormendy relations. Winds are even more effective if the smallest mass to be merged into a bulge is reduced to $3 \%$ of $M_{*}$, rather than our standard $0.1 M_{*}$. This is shown in Figure 6. The basic merger rate is the same, so the redshifts of assembly are not greatly altered. However because the bulge is being built of more, smaller, units, the buildup has less dispersion in time.

\section{Conclusions}

The bulge formation history is predicted here using the observed density of nearby (gasrich) galaxies with masses comparable to the eventual bulges. Further, we have argued that starburst winds will have a significant effect on the accreting gas. A straightforward assessment of our results is that the merger history appears to be roughly in accord with what is known about star formation histories and bulge ages. An additional step, not taken here, is to use these assembly histories to predict the color distribution of bulges as 

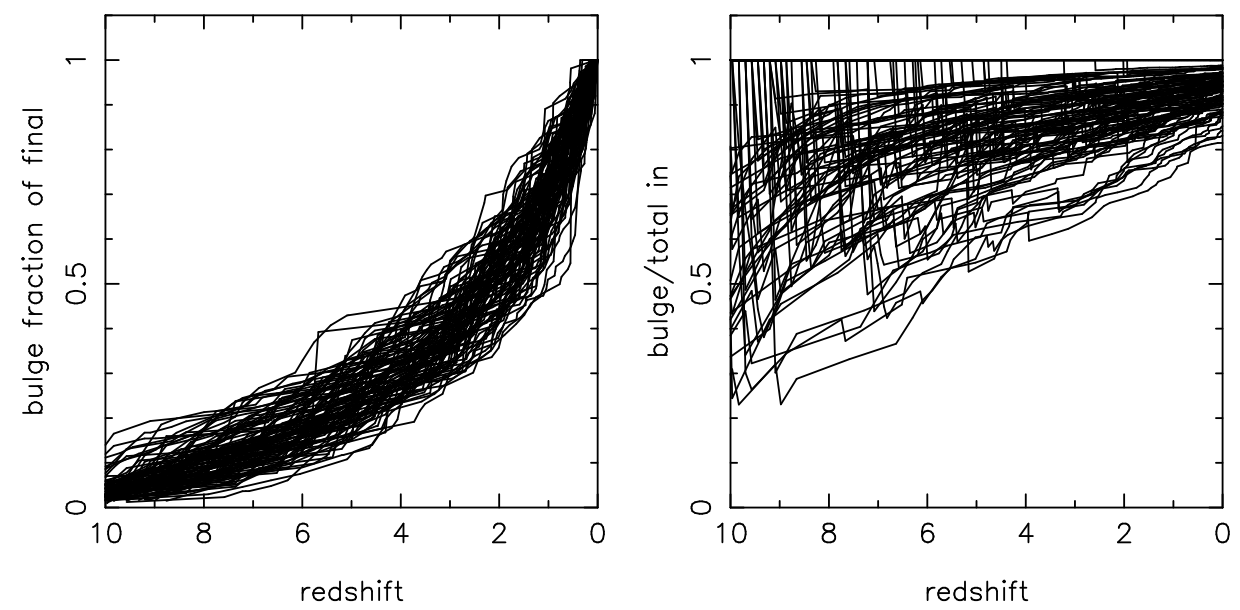

FiguRE 6. The assembly of a bulge built from pre-galactic objects with a minimum mass of $0.03 M_{*}$, rather than the standard $0.1 M_{*}$ and wind lifetime of $10^{7} \mathrm{yr}$. The left panel is the analog of Figure 2 and the right panel is the analog of Figure 3 . The assembly here is smoother and more affected by the starburst winds. Although there is less dispersion in formation times, the median $20 \%$ and $50 \%$ time are not significantly different from the standard model.

a function of redshift. These then become a simple but powerful test of the model. The attraction of the merger model is that it is based on observations, which now provide a very basic measure over the redshift zero to four range, although the masses of the incoming objects are not well quantified at the highest redshifts. Beyond redshift four, this model is likely becoming less reliable, since the assumption that the pregalactic fragments are in place has no basis in observation and even in a low density Universe, the Press-Schechter approach would indicate that the halos in which these reside are becoming less numerous. Furthermore the gas content probably evolves with redshift. An attraction of this approach is that the need for additional physical parameters can be driven by observation.

The relevance for bulges of the strong starburst winds that we have advocated is far less clear at this stage. The attraction of the idea is that it promotes the development of the Kormendy relations in a self-gravitating system. The tests of this model will require fairly detailed observations of bulges at high redshifts, particularly concentrating on the heavily reddened objects in which starbursts generally occur. The presence of starburst winds and their affects can potentially be detected in optical emission lines, X-ray observations of hot gas (e.g. Heckman et al. 1999) and ultimately in resolved observations of the molecular gas (e.g. Frayer et al. 1999) which is known to exist.

This research was supported by NSERC of Canada. I thank the Carnegie Observatories, Pasadena, for their hospitality during the time when this work was initiated.

\section{REFERENCES}

Aarseth, S. J. \& Fall, S. M. 1980, ApJ, 236, 43

Adelberger, K. L., Steidel, C. C., Giavalisco, M., Dickinson, M., Pettini, M., \& Kellogg, M. 1998, ApJ, 505, 18

Barnes, J. E. \& Hernquist, L. 1992, ARAA, 30, 705 
Baugh, C. M, Benson, A. J., Cole, S., Frenk, C. S., \& Lacey, C. G. 1998, MNRAS, submitted astro-ph/9811222

Carlberg, R. G. \& Couchman, H. M. P. 1989, ApJ, 340, 47

Carlberg, R. G. 1991, ApJ, 367, 385

Carlberg, R. G., Yee, H. K. C., Morris, S. L., Lin, H., Sawicki, M., Wirth, G., Patton, D., Shepherd, C. W., Ellingson, E., Schade, D., Pritchet, C. J., \& Hartwick, F. D. A. 1998, Phil. Trans. Roy. Soc., in press.

Chevalier, R. A. \& Clegg, A. W. 1985, Nature, 317, 44

Colin, P., Carlberg, R. G., \& Couchman, H. M. P. 1997, ApJ, 390, 1

Davies, R. L., Efstathiou, G., Fall, S. M., Illingworth, G. \& Schechter, P. L. 1983, ApJ, 266, 41

Davis, M. \& Peebles, P. J. E. 1983, ApJ, 267, 465

De Jong, R. S. 1996, A\&Ap, 313, 45

Dubinski, J., Mihos, J. C., \& Hernquist, L. 1999, ApJ submitted astro-ph/9902217)

Frayer, D. T., et al. 1999, ApJL, 514, L13

Giavalisco, M., Steidel, C. C., Adelberger, K. L., Dickinson, M. E., Pettini, M., \& Kellogg, M. 1998, ApJ, 503, 543

Heckman, T. M., Armus, L., Weaver, K. A., \& Wang, J. 1999, ApJ, in press (astro-ph/9812317)

Ikeuchi, S. \& Norman, C. A. 1991, ApJ, 375, 479

Kennicutt, R. C. , Jr. 1998, ApJ, 498, 541

Kormendy, J. 1977, ApJ, 218, 333

Lehnert, M. D. \& Heckman, T. M. 1996, ApJ, 472, 546

Loveday, J., Maddox, S. J., Efstathiou, G. \& Peterson, B. A. 1995, ApJ, 442, 457

Marzke, R. O., Geller, M. J., Da Costa, L. N. \& Huchra, J. P. 1995, AJ, 110, 477

Nulsen, P. E. J. 1982, MNRAS, 198, 1007

Pahre, M. A., Djorgovski, S. G. \& De Carvalho, R. R. 1995, ApJL, 453, L17

Patton, D. R., Marzke, R. O., Carlberg, R. G., Pritchet, C. J., \& da Costa, L. N. 1999, ApJ, to be submitted.

Patton, D. R., Carlberg, R. G., Marzke, R. O., Yee, H. K. C., Lin, H., Morris, S. L., Sawicki, M., Wirth, G. D., Shepherd, C. W., Ellingson, E., Schade, D., \& Pritchet, C. J., 1999, ApJ, to be submitted.

Peebles, P. J. E. 1980, Large Scale Structure of the Universe (Princeton University Press: Princeton)

Steidel, C. C.. Adelberger. K. L., Giavalisco, M., Dickinson, M, \& Pettini, M. 1999, ApJ, in press (astro-ph/9811399)

Toomre, A. \& Toomre, J. 1972, ApJ, 178, 623

Toomre, A. 1977, in Evolution of Galaxies and Stellar Populations, ed. B. M. Tinsley \& R. B. Larson, (Yale Observatory: New Haven) p. 401

Wyse, R. F. G., Gilmore, G. \& Franx, M. 1997, ARAA, 35, 637 